\title{
The Multicomponent Working Memory Model, Attention, and Long-term Memory in Multimedia Learning: A Comment on Schweppe and Rummer (2014)
}

\author{
Alexander Soemer \\ Published online: 6 March 2015 \\ (C) Springer Science+Business Media New York 2015
}

Multimedia learning theories rely on findings from different areas of fundamental psychological research. Working memory is undoubtedly a particularly important concept for these theories. However, the application of findings from fundamental research to multimedia learning is not as straightforward as it might seem at first glance, and misinterpretations are pervasive in the literature.

The recent article of Schweppe and Rummer (2014) represents an important contribution in this respect. Their paper outlines assumptions in Baddeley and Hitch's (1974) multicomponent working model and discusses the implementation of these assumptions in popular multimedia learning theories such as the cognitive theory of multimedia learning (CTML; Mayer 2009) and cognitive load theory (CLT; Sweller et al. 2011). The authors then continue arguing that the multicomponent model conceives working memory as structurally separate from long-term memory, and that this assumed separation has drawbacks with regard to explaining a number of effects in multimedia studies. As an alternative foundation for multimedia learning theories, they propose Cowan's (1999) embedded-processes model.

While this effort should be highly valued, the present commentary argues (a) that Schweppe and Rummer's rejection of the multicomponent model as a basis for multimedia theories is based on a misinterpretation of the relationship between working memory and long-term memory in the multicomponent model, (b) that the multicomponent model and the embeddedprocesses model can be seen as complementary approaches, and (c) that a complete theory of multimedia learning needs to fully integrate both central executive (i.e., attentional) and functionally specialized (e.g., phonological and visuospatial) mechanisms.

\section{The Relationship Between Working Memory and Long-term Memory in Baddeley's Model}

Schweppe and Rummer state that the multicomponent model "conceives of working memory as a cognitive system separate from long-term memory" (p.286). The crucial question, however, is whether this separation is structural or functional. Schweppe and Rummer seem

\footnotetext{
A. Soemer $(\bowtie)$

Graduate School of Education, Kyoto University, Yoshida-honmachi, Sakyo-ku, Kyoto 606-8501, Japan e-mail: alexander@soemurai.de
} 
to adopt the former view by stating that Baddeley's (2000) modification of the original model "maintains the structural separation of working memory and long-term memory." However, structural separation is nowhere asserted in Baddeley (2000) and, in fact, constitutes a frequent misunderstanding of the model. Baddeley and Logie (1999, p.47) explicitly state that they "[...] believe that working memory and LTM comprise two functionally separable cognitive systems" (italics added by author). Baddeley (2012) adds that "working memory involves the activation of many areas of the brain that involve LTM" (p.18). Apparently, the multicomponent model is a functional not a structural working memory model, contrary to Schweppe and Rummer's characterization and contrary to what is assumed in multimedia learning theories. Functional separation, however, is entirely compatible with the view that activated long-term memory representations form a part of working memory. The multicomponent model separates working memory functionally from long-term memory in order to account for functionality that goes beyond the mere activation of long-term memory traces, for example, functionality necessary for learning new information (Baddeley et al. 1998).

A related point concerns the relationship between the multicomponent model's subcomponents and long-term memory. Although earlier versions of the model assumed no close relationship, this view was modified in the 1990s, largely based on work demonstrating heavy involvement of phonological loop in verbal learning (Baddeley et al. 1998). Not only does this work predate the introduction of the episodic buffer, even Baddeley's (2000) modified version of the model clearly shows direct connections between the subcomponents and long-term memory that do not involve the buffer (Baddeley 2000, Fig. 1b). In other words, contrary to Schweppe and Rummer's claim (p. 288), long-term memory contributions to phonological and visuospatial processing are well established within the framework of the multicomponent model.

\section{The Complementary Relationship Between Baddeley's Model and Cowan's Model}

Until more recently (e.g., Baddeley et al. 2011), the precise layout and function of the central executive were underspecified within the framework of the multicomponent model. Cowan's embedded-processes model (Cowan 1999), on the other hand, filled this gap by detailing out a single central executive (i.e., attentional) mechanism that is able to (re)activate any long-term memory trace irrespective of its content. Cowan has argued for the importance of such a general mechanism based on experiments showing that - under certain conditions - working memory capacity estimates do not differ much between single and dual span tasks with verbal and visual materials (e.g., Saults and Cowan 2007).

This, however, is not to say that Cowan's model rejects the notion that humans can make use of functionally specialized processes that demand minimal central executive attention such as articulatory rehearsal for the case of verbal materials. Cowan previously simply tended to regard these processes as not reflecting the "real" working memory, making the difference between Baddeley's and Cowan's models, to a large part, a difference in focus and terminology. Note that this view has recently been modified based on newer experiments. Cowan et al. (2014) now suggest that "[...] the notion of the focus of attention as a central holding area is too simplistic to account for a wide range of experimental results involving the combination of verbal and visual stimuli" (p.25) and outline a working memory model made up of both specialized verbal and visual working memory resources ("peripheral resources" in their terminology), as well as attentional resources ("central resources" in their terminology). 
In sum, although the multicomponent model and the embedded-processes model seem to be very different at a superficial level, they are, in fact, both functional working memory models that tackle the same problem from different sides.

\section{Implications for Multimedia Learning Theories}

Relatively small between-task interference in comparison to within-task interference in dualtask studies with verbal and visuospatial materials continues to constitute a strong argument for the functional separation of phonological and (visuo)spatial working memory. Furthermore, the multicomponent model is open to the addition or further fractionation of components, provided there is enough evidence. For example, recent experiments by Williamson et al. (2010) suggest different stores for auditory and verbal materials. Others have corroborated such a fractionation by showing that maintenance of nonverbal sounds in working memory may involve processes similar to auditory imagery but not articulatory rehearsal (Soemer and Saito 2015).

Such kinds of functional specialization are not paid attention to in the embedded-processes model. Nevertheless, they are relevant for theories of multimedia learning. Both CLT and CTML are based on the assumption that working memory is critical for learning but highly capacity-limited. In CLT, working memory capacity determines the amount of cognitive resources available for dealing with intrinsic, extraneous, and germane load. Furthermore, in both CTL and CTML, the visual and the auditory modalities have their own capacity limitations. ${ }^{1}$ It is not difficult to see that the question of whether there is a single capacity limit (as assumed in the embedded-processes model), or multiple capacity limits originating from functional specialization (as assumed in the multicomponent model), is highly relevant for the design of multimedia materials.

On the other hand, Schweppe and Rummer are certainly correct in encouraging multimedia researchers to pay more attention to the central executive side of working memory (Miyake et al. 2000). Firstly, due to the large amount of information in multimedia learning materials, it is certain that functionally specialized processing will not be sufficient and that the central executive will be heavily involved in the control of storage and maintenance activities. Secondly, as learning is more than only storage and maintenance of incoming information, incorporating findings from research on central executive functions such as updating (replacing no longer relevant information in working memory with newer relevant information) and task switching (shifting between two or more to-be-performed mental operations) may prove to be fruitful for rethinking processing assumptions in multimedia learning theories (e.g., selection, organization, and integration of information in CTML). Thus, paying more attention to the central executive may enhance theorizing in multimedia research and lead to the formulation of new instructional principles.

To summarize, rather than switching from a multicomponent view to an embeddedprocesses view, the present commentary suggests that a fruitful revision/extension of multimedia theories will have (1) to take into account functionally specialized components, (2) to correct misinterpretations regarding the multicomponent model, and (3) to incorporate findings from research on central executive functions.

\footnotetext{
${ }^{1}$ Note that the multicomponent model distinguishes between a visuospatial and a verbal component, not a visual and an auditory component. Multimedia learning theories deviate from the multicomponent model at this point.
} 


\section{References}

Baddeley, A. D. (2000). The episodic buffer: a new component of working memory? Trends in Cognitive Sciences, 4, 417-423.

Baddeley, A. D. (2012). Working memory: theories, models, and controversies. Annual Review of Psychology, $63,1-29$.

Baddeley, A. D., \& Hitch, G. J. (1974). Working memory. In G. Bower, The psychology of learning and motivation: advances in research and theory (Vol. 8, 47-89). New York: Academic Press.

Baddeley, A. D., \& Logie, R. (1999). Working memory: the multiple-component model. In A. Miyake \& P. Shah (Eds.), Models of working memory: mechanisms of active maintenance and executive control. Cambridge: Cambridge University Press.

Baddeley, A. D., Gathercole, S., \& Papagno, C. (1998). The phonological loop as a language learning device. Psychological Review, 105, 158-173.

Baddeley, A. D., Allen, R. J., \& Hitch, G. J. (2011). Binding in visual working memory: the role of the episodic buffer. Neuropsychologia, 49, 1393-1400.

Cowan, N. (1999). An embedded-processes model of working memory. In A. Miyake \& P. Shah (Eds.), Models of working memory: mechanisms of active maintenance and executive control. Cambridge: Cambridge University Press.

Cowan, N., Saults, J. S., \& Blume, C. L. (2014). Central and peripheral components of working memory storage. Journal of Experimental Psychology: General, 143, 1806-1836.

Mayer, R. E. (2009). Multimedia learning. Cambridge: Cambridge University Press.

Miyake, A., Friedman, N. P., Emerson, M. J., Witzki, A. H., Howerter, A., \& Wager, T. D. (2000). The unity and diversity of executive functions and their contributions to complex "frontal lobe" tasks: a latent variable analysis. Cognitive Psychology, 41, 49-100.

Saults, J. S., \& Cowan, N. (2007). A central capacity limit to the simultaneous storage of visual and auditory arrays in working memory. Journal of Experimental Psychology: General, 136, 663-684.

Schweppe, J., \& Rummer, R. (2014). Attention, working memory, and long-term memory in multimedia learning: an integrated perspective based on process models of working memory. Educational Psychology Review, 26, 285-306.

Soemer, A. \& Saito, S. (2015). Maintenance of auditory information in working memory. Manuscript submitted for publication.

Sweller, J., Ayres, P., \& Kalyuga, S. (2011). Cognitive load theory. New York: Springer.

Williamson, V. J., Baddeley, A. D., \& Hitch, G. J. (2010). Musicians' and nonmusicians' short-term memory for verbal and musical sequences: comparing phonological similarity and pitch proximity. Memory \& Cognition, 38, 163-175. 